\title{
Conception of a Coplanar Circulator Using a Triangular Structure for Application at X-Band Frequency
}

\author{
Rahmouna El-Bouslemti ${ }^{1}$, Faouzi Salah-Belkhodja ${ }^{1}$, Didier Vincent ${ }^{2}$, \\ Rafah Naoum ${ }^{1}$ \\ ${ }^{I}$ (Telecommunications and Digital Signal Processing Laboratory, Djillali Liabes University, Algeria) \\ ${ }^{2}$ (Télécom Claude Chappe (LT2C) Laboratory, University of Saint-Etienne, France)
}

Abstract : The new triangular structure of a coplanar circulator is proposed and studied numerically in this work. The structure under study is made from a massive ferrite film (1mm thick). The HFSS is used to simulate the circulator and to check the non-reciprocal characteristics at operating frequency. An insertion loss of $-0.46 \mathrm{~dB}$ and isolation of $-29.88 \mathrm{~dB}$ has been achieved by optimizing the structure at $10.6 \mathrm{GHz}$.

Keywords: Circulator, CPW, ferrite, microwave, non reciprocity, S-parameters, triangular stricture

\section{INTRODUCTION}

In recent years, the increasing development of microwave applications required the optimization of devices to meet the requirements of the telecommunications market.

The devices must therefore be developed miniaturized, low-cost products, while operating at higher frequency ranges. The device most commonly used in these systems is a microstrip Y-junction circulator. The operating principle of a non-reciprocal Y- circulator based on a ferrite has been described by Bosma since 1962 [1]. In this analysis the Polder tensor is used to describe the tensor permeability of the ferrite. Though, a circulator with coplanar waveguide (CPW) structure is well suited to microwave integrated circuits because line and ground (GND) are located in the same plane and are easily patterned.

Wen [2] is the first who has studied and developed the theory of coplanar waveguide (CPW). After that; Ogasawara [3] has showed and has confirmed that the Y-junction circulators with CPW successfully operated at certain frequencies. Many researchers have been studied and realized the circulators with a CPW structure. Zahwe $[4,5]$ was well studied and developed this device. Boyajian [6] was proposed a ciculator with Hexaferrite nanocomposite. In our previous paper, a new circulator having a triangular form with a $\mathrm{CPW}$ structure was proposed and its transmission characteristics were analyzed using an Ansoft HFSS. The study of a such circulator is based on the Helszajn's model $[8,9]$ for stripline structure. Later, Zahwe studied this type of circulation in 2007[10]. This type of circulator has an operation similar to that of circulator stripline. The main purpose is to create a miniature circulator with YIG (ferrite of $1 \mathrm{~mm}$ thick). The component that will be used to develop must be having a low insertion loss, a high isolation and low reflection levels at all ports.

\section{ANAlytical Modeling Of STRIPline Circulator}

In this work we have studied the particular case of coplanar circulators having a central conductor triangular in shape and based on the studies of J. Helszajin [8] and Bosma [7]. This study helps to understand the operation and the good working of the circulator. the relation:

From the theoretical results obtained by Bosma [7], the internal bias field layer of ferrite is defined by

$$
\mathrm{H}_{\mathrm{i}}=\sqrt{\frac{\lambda}{\sqrt{3} \mathrm{w}} \mathrm{H}_{0}-4 \pi \mathrm{M}_{\mathrm{s}}}-4 \pi \mathrm{M}_{\mathrm{s}}
$$

Where $\lambda$ is the free space wavelength, $w$ is the radius of the central conductor, $M_{s}$ is the saturation magnetisation and $\mathrm{H}_{0}$ is the external static field is defined by:

$$
\mathrm{H}_{0}=\frac{\omega}{\gamma}
$$

With $\gamma, \omega$ respectively represent the gyromagnetic factor, the angular frequency. The Calculation of the coordinates of the center conductor of the circulator (see Fig.1) was calculated from the following formulas from [7]:

$$
\mathrm{R} / \lambda=\frac{\chi_{1,1}}{2 \pi \sqrt{\epsilon}} \sqrt{\frac{\mathrm{H}_{\mathrm{i}}}{\mathrm{H}_{\mathrm{i}}+4 \pi \mathrm{M}_{s}}} \quad \text { and } \quad \psi=\frac{1}{\sqrt{3}} \frac{\pi(\mathrm{k} / \mu)}{1.84 \sqrt{\mu_{\mathrm{eff}} / \varepsilon}}
$$


Where $\mathrm{R}$ is the radius of the ferrite disk used in the model of Bosma [1] and $\psi$ is a stripline width angle, it is defined by:

$$
\psi=\sin (W / 2 R)^{-1}
$$

According to results cited in [8]:

$$
r=\frac{A}{2 \sqrt{3}}
$$

$\mathrm{r}$ either represents the inscribed radius defined by the triangular resonator, and $\mathrm{A}$ is the length of the side of the inner triangular conductor.

Or the actual radius of the ferrite disk [8]

$$
r=R
$$

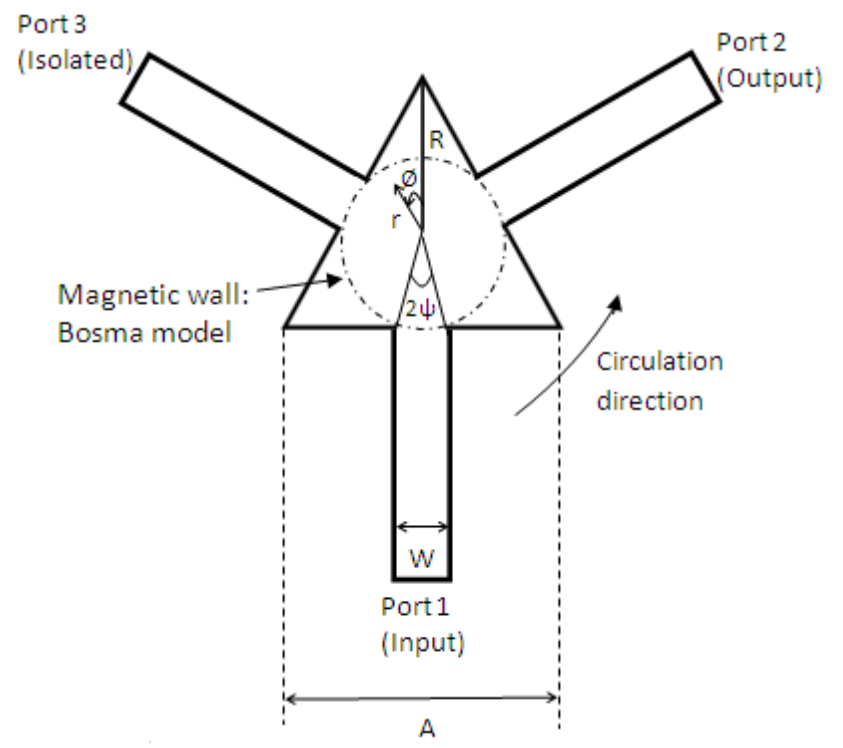

Fig. 1 Coordinates of the center conductor of the triangular circulator

We can calculate the size of A from this equation [5]:

$$
\mathrm{kA}=4 \pi / 3
$$

$\mathrm{k}$ is the wave number in the medium and is defined by:

$$
\mathrm{k}=\frac{2 \pi}{\lambda} \sqrt{\epsilon_{\mathrm{f}} \mu_{\mathrm{eff}}} \quad \text { and } \quad \mu_{\mathrm{eff}}=\frac{\mu^{2}-\mathrm{k}^{2}}{\mu}
$$

Where $\epsilon_{\mathrm{f}}$ is the relative dielectric constant, $\mu_{\mathrm{eff}}$ is the effective permeability of the ferrite calculated from the model of Polder. $k$ et $\mu$ are the elements of the tensor Polder ferrite.

From measurements of the resonant frequency for the first mode, authors in [9] found that the edges of the resonator are not really perfect magnetic walls. Zahwe was defined the effective value of the triangle side $\mathrm{A}_{\text {eff }}$ as follows [10]:

$$
A_{\text {eff }}=A+\frac{h}{4}
$$

Where $\mathrm{h}$ is the thickness of the ferrite layer.

\section{Design OF Triangular CirCulator}

Figure 2 shows the circulator designed, which has a hexagonal structure coplanar (CPW: coplanar waveguide). The central part of our device (see Fig.1) has a triangular configuration. This Y-junction circulator has three accesses oriented at $120^{\circ}$ relative to each other.

The metallization (ground plane (GND) of $1 \mu \mathrm{m}$ thickness is made of gold. A ground plane (GND) in the form of a triangular thin film is placed at the center of the junction on the ferrite. This lower ground plane provides the signal transition from access lines (horizontally) to central conductive portion triangular lines (horizontal) [11] (see Fig.2). To ensure proper operation of the device, a magnetic bias field $\left(\mathrm{H}_{\mathrm{i}}\right)$ must be applied in a direction perpendicular to the solid layer of ferrite to assure the non-reciprocity of the circulator; the signal is transmitted in one direction and attenuated in the reverse direction. 


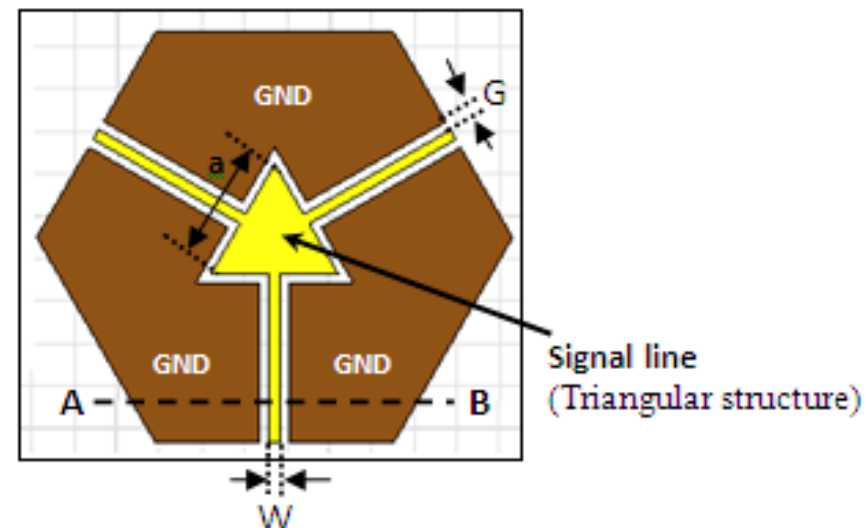

(a)
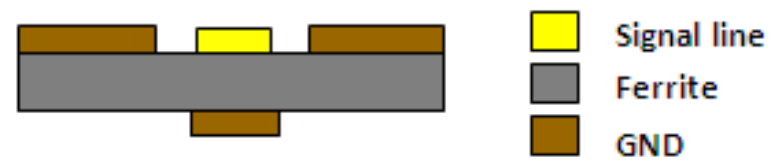

(b)

Fig.2 Structure of a triangular circulator with CPW technology. (a) top view, (b) cross sectional view $\mathrm{AB}$

The thickness of the ferrite layer is $\mathrm{h}=1 \mathrm{~mm}$. The magnetic material (ferrite) is defined by the following features: a relative permittivity $\varepsilon r=15.3$, a dielectric loss tangent $\tan \delta=10^{-2}$, a ferromagnetic resonance (FMR) line width $\Delta \mathrm{H}=100 \mathrm{Oe}$ and a saturation magnetization $\mathrm{M}_{\mathrm{s}}=178 \mathrm{mT}$._An internal magnetic field polarization $\mathrm{Hi}=477 \mathrm{KA} / \mathrm{m}$ was applied in a direction perpendicular to the ferrite layer to gets its special nonreciprocal properties from this magnetostatic bias field that is assumed to be uniform in magnitude and direction (see Fig. 3 )._The metallization (signal line and GND) with the gold has a relative permeability $\mu=0.99996$ and a conductivity $\sigma=41.10^{6} \mathrm{~S} / \mathrm{m}$ at $9.4 \mathrm{GHz}$.

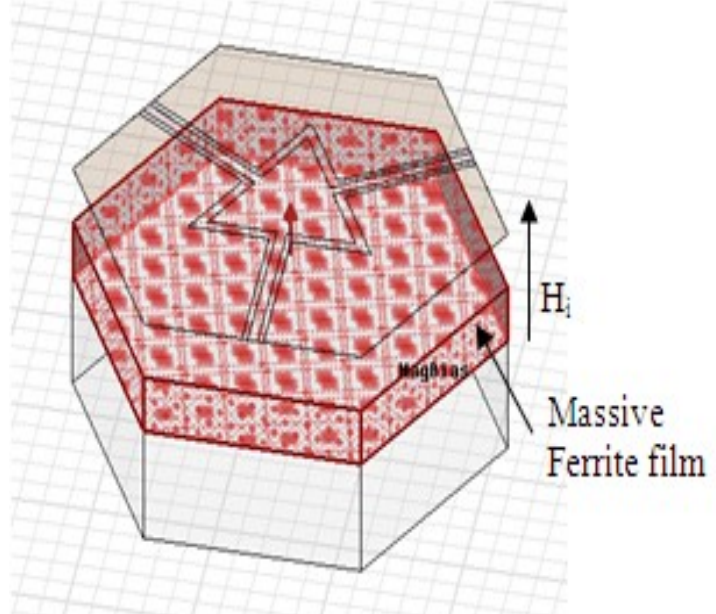

Fig.3 The perpendicular magnetic excitation in the ferrite

IV.

NUMERICAL STUDY AND DISCUSSION

Several simulations using the Ansoft HFSS simulator based on the method of finite elements are made to study the effect of changes in various parameters on the performance of the device. After optimization of the parameters, the dimensions of our circulator were defined as follows:

$\mathrm{A}=1.2 \mathrm{~mm}$ (the side of the inner central triangle), $\mathrm{W}=0.14 \mathrm{~mm}$ (the width of signal line), $\mathrm{G}=0.12$ $\mathrm{mm}$ (a signal Line-to-GND spacing). The resulting characteristic impedance is equal to $46 \Omega$.

To start the simulation, it is necessary to excite the structure in HFSS simulator. We use the WavePorts that are specified for the excitation signal is not fully enclosed in the slot and extend into the air above the ferrite (see Fig.4). The wave port is aligned with the CPW to provide a window that couples the model device to the external world. In the HFSS the CPW is surrounded by a sufficient distance of the airbox of the line. 


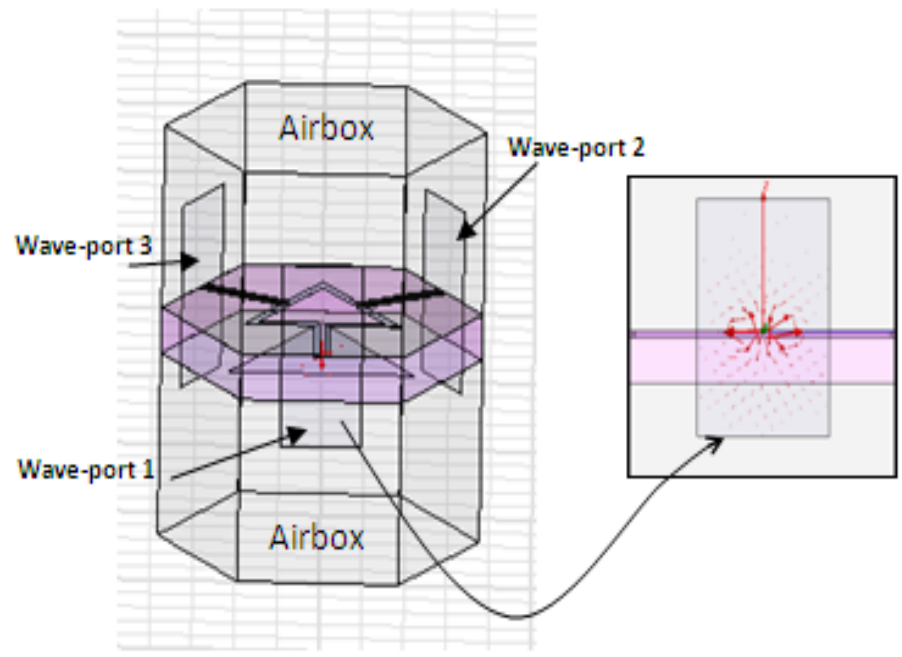

Fig. 4 Wave-Port: Configuration of the electrical signal

The three-dimensional simulation gives the S-parameters, shown in Fig.5, which assess the performance of the circulator. The signal is propagated from one port to another, with insertion losses of $0.46 \mathrm{~dB}$ presented by $\mathrm{S}_{21}$, in the opposite direction the signal is blocked with an isolation loss $\mathrm{S}_{31}=-21.22 \mathrm{~dB}$ and $\mathrm{S}_{12}=-29.88 \mathrm{~dB}$. These values are satisfactory and show the proper functioning of the circulator. The effect of non-reciprocal traffic is observed at a frequency of $10.6 \mathrm{GHz}$.

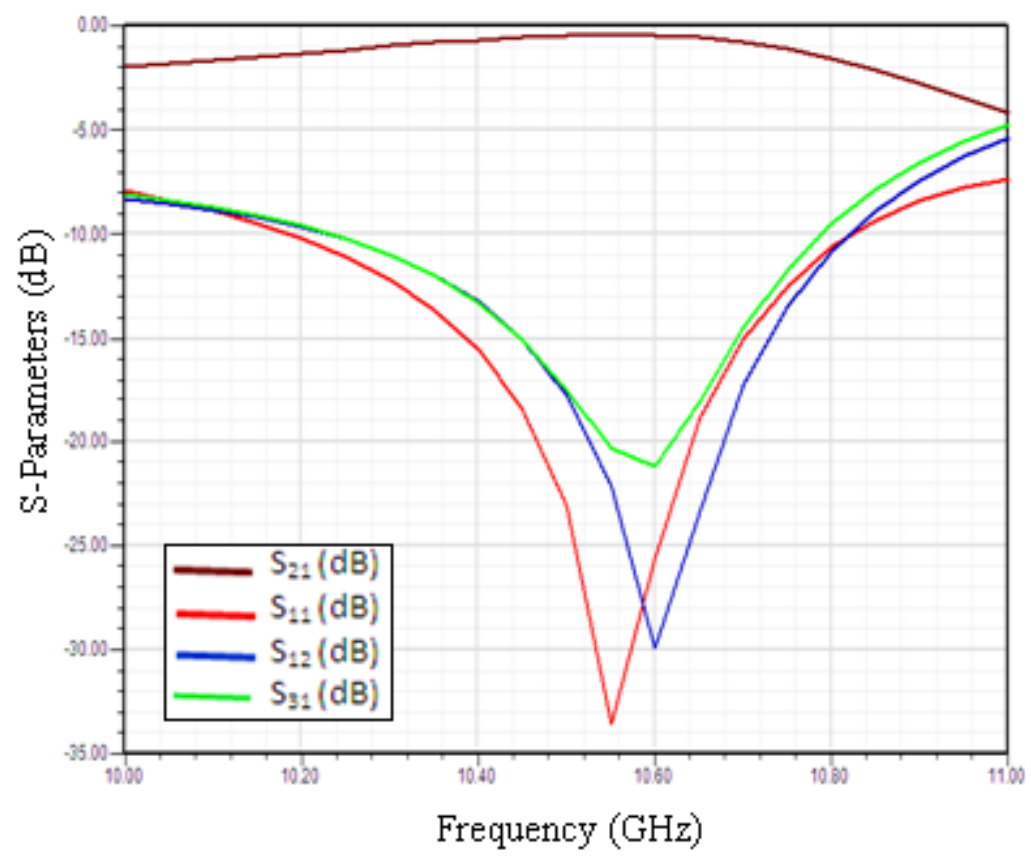

Fig.5 Evolution of S-parameters of a triangular coplanar circulator

\subsection{The Effect of Variation of the Gap G on the S-parameters}

In order to investigate the sensitivity of triangular circulator performance towards the gap $\mathrm{G}$ (spacing between the GND plane and the signal line), we will calculate the $S_{11}$ (the return loss), $S_{21}$ (the insertion loss) and $\mathrm{S}_{31}$ (isolation).

The parameters $\mathrm{G}$ varies from 90 to $180 \mu \mathrm{m}$ keeping a W/G report as the resulting impedance of access remains close to $50 \Omega$. The simulation results obtained are presented in Fig.6. From these results, it appears that S-parameters vary irregularly with the slot $G$, The device gets a better performance for $G=120 \mu \mathrm{m}$ with $\mathrm{S}_{21}=-0.46 \mathrm{~dB}$. The insertion losses are very low. Apart from these values; the insertion loss also increase of about $1.6 \mathrm{~dB}$ and the return loss and isolation decreases until the disappearance of non-reciprocal effect of beyond $180 \mu \mathrm{m}$. Consequently, the slot $\mathrm{G}$ of our circulator is set to be $120 \mathrm{um}$. 


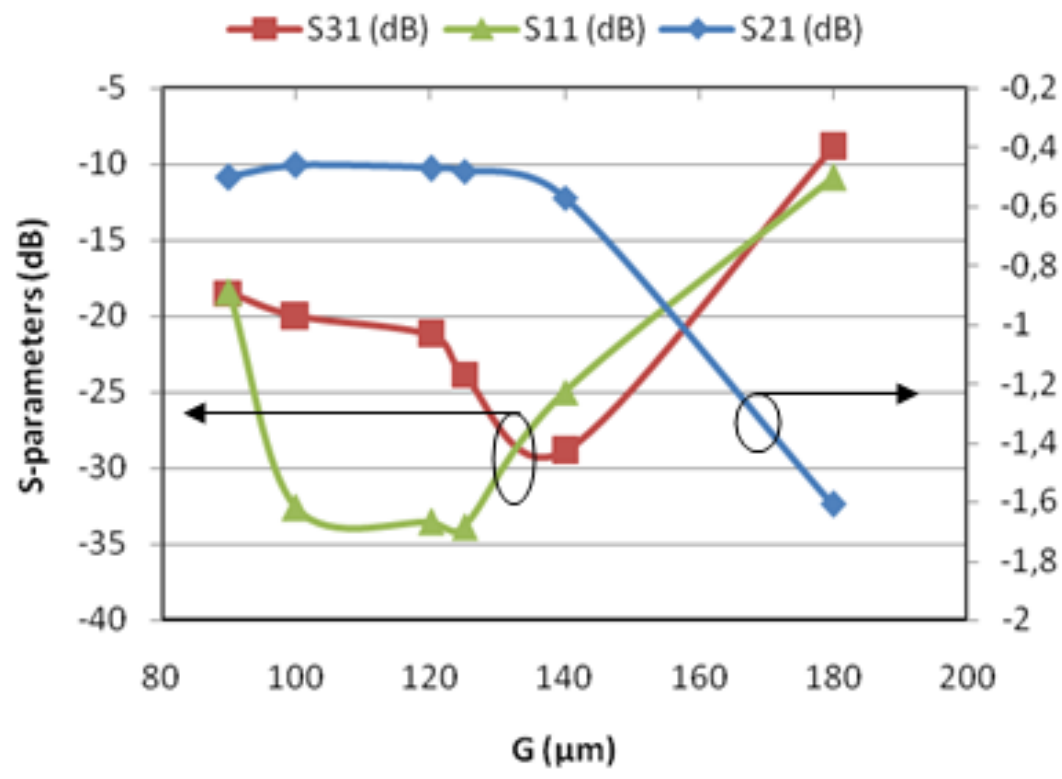

Fig.6 Dependence of S-parameters on the G (a signal Line-to-GND spacing)

\subsection{Effect of Ground Plane Lower}

\subsubsection{Variation of the Radius of the Triangular Ground Plan Lower $R_{G}$}

The effect of the $\mathrm{R}_{\mathrm{G}}$ parameter (radius of triangular GND) on transmission characteristic, such as insertion loss $S_{21}$ and non-reciprocity $S_{12}$ is investigated. This parameter was calculated from (5) that shows the geometric relationship between the side and the radius of the circumcircle of a triangle (see Fig.1).

The results presented in the figure below shows that the gap $R_{G}$ varies from $2.13 \mathrm{~mm}$ to $2.63 \mathrm{~mm}$. The variation of the radius of the ground plane induces a variation in insertion loss of the order of $0.89 \mathrm{~dB}$; this quantity is close to $1 \mathrm{~dB}$ and it is not negligible for the radii less than $2.13 \mathrm{~mm}$. The frequency was influenced by the variation of $\mathrm{R}_{\mathrm{G}}$, the circulation frequency is $10.4 \mathrm{GHz}$ for $\mathrm{R}_{\mathrm{G}}=2.13 \mathrm{~mm}$ and it becomes $11 \mathrm{GHz}$ for $\mathrm{R}_{\mathrm{G}}=2.63 \mathrm{~mm}$. We notice that the variation of radius of triangular GND has a great impact on the isolation loss which reaches $-14 \mathrm{~dB}$ when the radius overflows too much from the upper triangular conductor; however the return losses varies in an irregular manner. The best results are obtained for a radius $R_{G}=2.53 \mathrm{~m}$ $\left(\mathrm{S}_{21}=-0.46 \mathrm{~dB}\right.$ and $\mathrm{S}_{12}=-29.88 \mathrm{~dB}$ at $\left.10.6 \mathrm{GHz}\right)$.

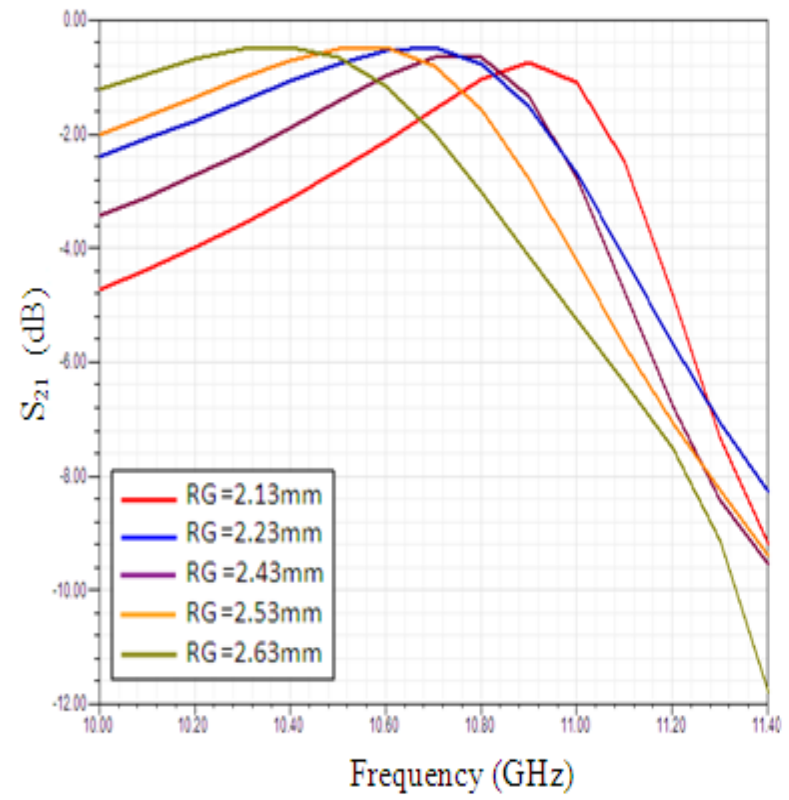

(a)

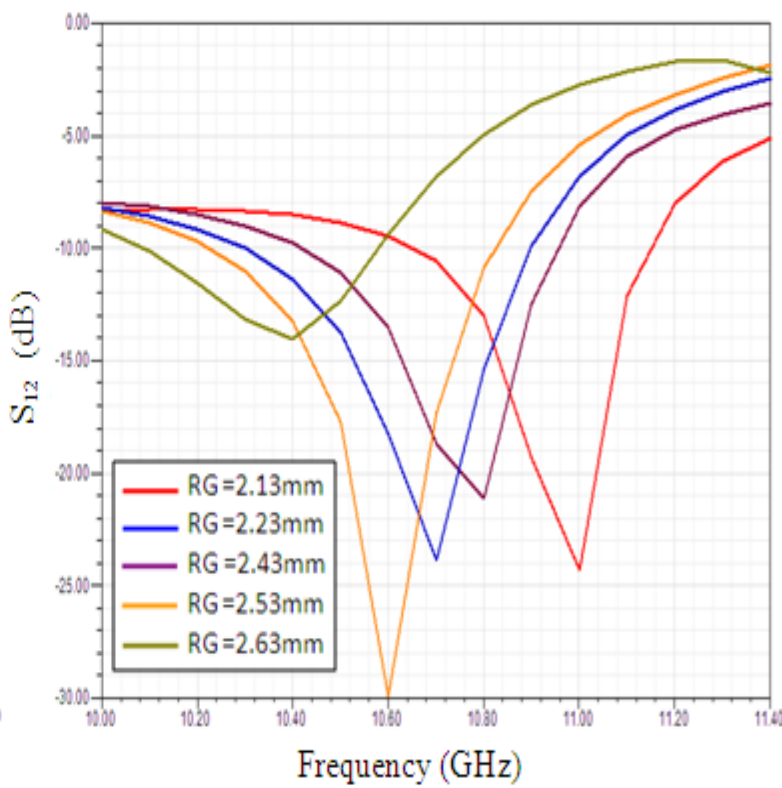

(b)

Fig.7 Dependence of S-parameters on the $\mathrm{G}_{1}$ (a signal Line-to-GND spacing). (a) Insertion loss (b) Isolation 
4.2.2 Without the Triangular Ground Plane Lower

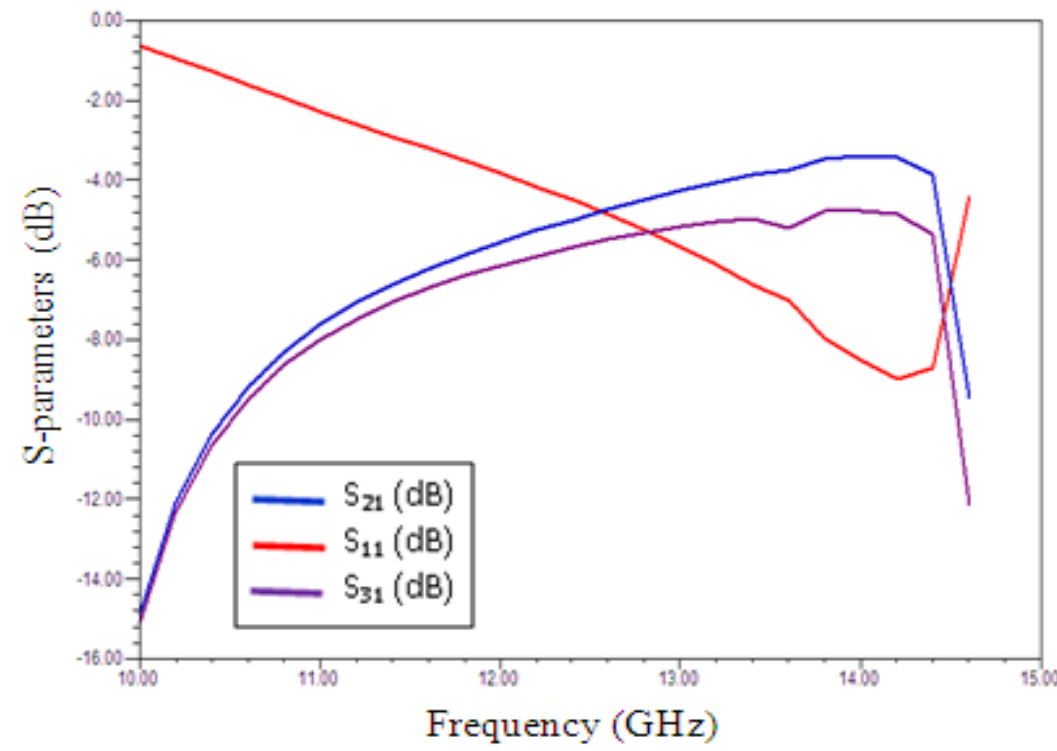

Fig.8 S-parameter of a triangular circulator with which the GND lower is removed

We have removed the GND ground plane lower which is located above the ferrite in the structure presented in Fig. 1 to show its effect. The frequency characteristics of S-parameters for a circulator are reported on Fig. 8. The circulation Phenomenon has disappeared and the transmission is reciprocal, this ensures that the GND plane allows a better transition of the wave from the access lines (horizontally) to the circulator's central part (vertically) [11].

\subsection{Effects of the $\Delta \mathrm{H}$ on Transmission Characteristics}

We conducted a series of simulations to quantify the effect of the linewidth resonance $\Delta H$ (see Fig.9). The effect of the $\Delta H$ on insertion losses can be seen by setting the perfect conductor and the dielectric loss tangent to zero [12].

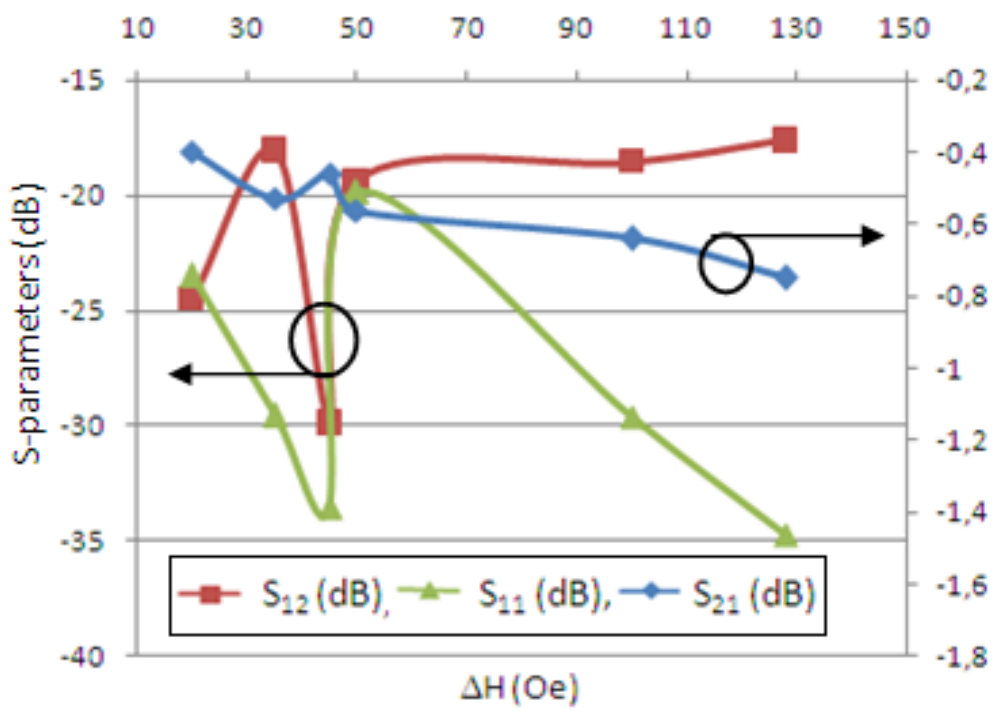

Fig.9 Dependence of $S$-parameters $\left(S_{21}, S 12, S 11\right)$ on the $\Delta H$ at $10 \mathrm{GHz}$

From the simulation results, the return loss and the isolation loss vary in an irregular manner. The absolute value of the insertion loss increase with $\Delta \mathrm{H}$. The best performance is obtained at the $\Delta \mathrm{H}=20 \mathrm{Oe}$, with the insertion loss is about $-0.40 \mathrm{~dB}$. The return loss and the isolation fluctuates with the $\Delta \mathrm{H}$. The change of $\Delta \mathrm{H}$ implies a variation of magnetic properties of ferrite which creates losses in the device. Benevent indicated that the losses are predominant, are due to the magnetic and dielectric losses and the conductor losses cab be considered as negligible [13]. With regard to the insertion losses, therefore for ensuring the proper functioning 
of the device it should be reduced losses that it must be low as possible; as a solution is to change the topology of circulator using a triangular central conductor [13].

\section{CONCLUSION}

This work presents a numerical study of a non-reciprocal circulator where the central part of the device has a triangular pattern, based on a magnetic material of ferrite of $1 \mathrm{~mm}$ thickness. The performance of the circulator has been studied numerically using Ansoft HFSS.

In addition, ZAHWE [10] studied a coplanar circulator with triangular shape structure using a massive YIG ferrite substrate of $1 \mathrm{~mm}$ thickness; access lines are connected to the apex of the triangle (principal conductor). This structure is different from our structure that is presented in this work, which allowed us to compare the two works. The following table provides the results of the comparison:

Table1: Comparison between our results and those published in [10].

\begin{tabular}{||l||c||c||c||c||}
\hline \multicolumn{1}{|c|}{ Authors } & $\begin{array}{c}\text { Insertion loss } \\
\mathbf{S}_{\mathbf{2 1}} \mathbf{( d B}\end{array}$ & $\begin{array}{c}\text { Isolation } \mathbf{S}_{\mathbf{3 1}} \\
\mathbf{( d B )}\end{array}$ & $\begin{array}{c}\text { Return loss } \\
\left.\mathbf{S}_{\mathbf{1 1}} \mathbf{( d B}\right)\end{array}$ & $\begin{array}{c}\text { The circulation } \\
\text { frequency }(\mathbf{G H z})\end{array}$ \\
\hline \hline ZAHWE & $-0.65 \mathrm{~dB}$ & $-15.03 \mathrm{~dB}$ & $-18.5 \mathrm{~dB}$ & $9.98 \mathrm{GHz}$ \\
\hline \hline El-BOUSLEMTI & $-0.46 \mathrm{~dB}$ & $-29.88 \mathrm{~dB}$ & $-33.57 \mathrm{~dB}$ & $10.6 \mathrm{GHz}$ \\
\hline
\end{tabular}

Comparing these two studies we conclude that we have obtained better results and better performance of the circulator. As such, we can say that it would be possible to achieve miniaturization and as a circulator for a massive ferrite layer of about $1 \mathrm{~mm}$.

\section{REFERENCES}

[1] H. Bosma, On the principle of stripline circulation, Proc. of The Institution of Electrical Engineers, London, Vol. 109, $N^{o} .21$, 1962, $137-146$.

[2] C. P. Wen, Coplanar waveguide, a surface strip transmission line suitable for a nonreciprocal gyromagnetic device applications, Proc. of G-MTT International Microwave Symposium, Dallas TX, USA, 1969, 110-115.

[3] K. Oshiro, H. Mikami, S. Fujii, T. Tanaka, H. Fujimori, M. Matsuura, and S. Yamamoto, Fabrication of circulator with coplanar wave guide structure, IEEE Trans on Magnetics, Vol. 41, No. 10, 2005, pp. 3550-3552.

[4] O. Zahwe, B. Sauviac, B. A. Samad, J. P. Chatelon and J.-J. Rousseau, Numerical study of a circulator using YIG thin film with a coplanar structure, Progress In Electromagnetics Research C, Vol. 6, 2009, 193-207.

[5] O. Zahwe, B. Sauviac and J.-J. Rousseau, Fabrication and measerment of a coplanar circulator with $65 \mu \mathrm{m}$ thin film, Prog in Electromagnetics Research Letters, Vol. 8, 2009, pp. 35-41.

[6] T. Boyajian, D. Vincent, M. Le Berre and S. Neveu, Study of a coplanar circulator based on a barium Hexaferrite nanocomposite, PIERS ONLINE, Vol. 7, No. 3, 2011, 201-205.

[7] H. Bosma, On stripline Y-circulation at UHF, IEEE Transaction on Microwave Theory and Techniques, Vol. 12, 1964, 61-72.

[8] J. Helszain, Fabrication of very weakly and weakly magnetized microstrip circulators, IEEE Transaction on Microwave Theory and Techniques, Vol. 46, No. 5, 1998, 439-449.

[9] J. Helszain, D.S. JAMES and W. TERENCE NISBET, Circulators using planar triangular resonators, IEEE Transaction on Microwave Theory and Techniques, Vol MTT-27, No. 2, 1979, 188-193.

[10] O. Zahwe, B. Sauviac, J.P. Chtelon, A-S. Dehlinger, M. Leberre and S. Perrot, Towards a miniaturized circulator with magnetic thin film, Proc. 37th European Microwave Conference, Munich, 2007, 274-277.

[11] E. Benevent, T. Rouiller, B. Sauviac, V. Larrey, D. Vincent and A. Madelaine, Stripline Y-junction circulator using barium hexagonal ferrite thin films, Proc. of IEEE International Symposium on Industrial Electronics, 2004, 15-18.

[12] Y. Wang, B. Peng, W. Zhang and K. Tan, CPW Circulators with Barium Ferrite Thin Films, Journal of Electronic Science and Technology, Vol. 8, No. 4, 2010, 351-355.

[13] E. Benevent, V. Larrey, D. Vincent and A. S. Delinger, Losses' origins in a $40 \mathrm{GHz}$ stripline circulator with $10 \mu \mathrm{m}$ thick barium hexagonal ferrite films, Proc. 37th European Microwave Conf, Manchester, 2006, 208-211. 